\title{
Avaliação da adesão medicamentosa dos pacientes atendidos em uma unidade de saúde da família em uma capital do nordeste, Brasil: Um estudo transversal observacional descritivo
}

Evaluation of medication adherence of patients treated at a family health unit in a capital of the northeast, Brazil: A descriptive observational cross-sectional study

Evaluación de la adherencia a la medicación de pacientes atendidos en una unidad de salud familiar en una capital del noreste de Brasil: Un estudio descriptivo observacional transversal

Luciano Vasconcellos Pacheco ORCID: https://orcid.org/0000-0002-6210-3828 Universidade do Estado da Bahia, Brasil E-mail: lucianofcd@hotmail.com

Geraldo Bezerra da Silva Júnior ORCID: https://orcid.org/0000-0002-8971-0994 Universidade de Fortaleza, Brasil E-mail: geraldobezerrajr@yahoo.com.br

Maria Teresita Bendicho ORCID: https://orcid.org/0000-0001-8234-1199 Universidade do Estado da Bahia, Brasil E-mail: mtbendicho@gmail.com

Rosa Malena Fagundes Xavier ORCID: https://orcid.org/0000-0002-3203-8949 Universidade do Estado da Bahia, Brasil E-mail: rxavier@uneb.br

\begin{abstract}
Resumo
Objetivo: Avaliar a adesão medicamentosa na população atendida em uma Unidade de Saúde da Família em uma capital do nordeste do Brasil. Métodos: Foi realizado um estudo transversal, observacional, descritivo, com pacientes vinculados à saúde da família entre o período de janeiro de 2017 a janeiro de 2019 em Salvador, Bahia, Brasil. Foram compilados pelo software EPI INFO os dados dos prontuários dos pacientes referentes ao perfil epidemiológico e às doenças prevalentes. Os dados sobre a adesão medicamentosa foram avaliados mediante o questionário de MoriskyGreen. O software Micromedex ${ }^{\circledR}$ foi utilizado para verificação de potenciais interações medicamentosas. Resultados: A população foi constituída de 121 pessoas atendidas na unidade de saúde, predominantemente mulheres pardas (83,6\% e 46,3 respectivamente), com prevalência para hipertensão arterial $(65,7 \%)$ e diabetes mellitus (40,3\%). Apenas 37,3\% dos pacientes apresentaram máxima adesão medicamentosa, principalmente devido ao esquecimento e ao descuido ao usar o medicamento (41,8\%). 37,9\% dos pacientes afirmaram ter que arcar financeiramente com a parcialidade ou totalidade do tratamento, mesmo com baixo poder aquisitivo $(77,7 \%$ com 2 salários mínimos ou menos). A maioria dos medicamentos prescritos foram relacionados ao sistema cardiovascular e $20,9 \%$ das prescrições apresentavam potenciais interações medicamentosas. Conclusão: A adesão medicamentosa foi intermediária para uma população com baixa renda e escolaridade média $(52,2 \%)$, com alta prevalência de doenças que afetam o sistema cardiovascular.
\end{abstract}

Palavras-chave: Adesão à medicação; Doenças cardiovasculares; Atenção primária em saúde; Estratégia de saúde da família.

\footnotetext{
Abstract

Objective: To evaluate medication adherence in the population served at a Family Health Unit in a capital city in northeastern Brazil. Methods: A cross-sectional, observational, descriptive study was carried out with patients linked to family health from January 2017 to January 2019 in Salvador, Bahia, Brazil. EPI INFO software compiled data from patients' medical records regarding the epidemiological profile and prevalent diseases. Data on medication adherence were assessed using the Morisky-Green questionnaire. The Micromedex ${ }^{\circledR}$ software was used to verify potential drug interactions. Results: The population consisted of 121 people seen at the health unit, predominantly brown women (83.6\% and 46.3 respectively), with a prevalence of arterial hypertension $(65.7 \%)$ and diabetes mellitus (40.3\%). Only $37.3 \%$ of patients showed maximum medication adherence, mainly due to forgetfulness and
} 
carelessness when using the medication (41.8\%). 37.9\% of the patients stated that they had to pay financially for partial or total treatment, even with low purchasing power (77.7\% with 2 minimum wages or less). Most of the prescribed drugs were related to the cardiovascular system and $20.9 \%$ of the prescriptions had potential drug interactions. Conclusion: Medication adherence was intermediate for a population with low income and average education $(52.2 \%)$, with a high prevalence of diseases that affect the cardiovascular system.

Keywords: Medication adherence; Cardiovascular diseases; Primary health care; Family health strategy.

\section{Resumen}

Objetivo: Evaluar la adherencia a la medicación en la población atendida en una Unidad de Salud de la Familia en una ciudad capital del noreste de Brasil. Métodos: Se realizó un estudio descriptivo, observacional y transversal con pacientes vinculados a la salud familiar desde enero de 2017 a enero de 2019 en Salvador, Bahía, Brasil. El software EPI INFO recopiló datos de las historias clínicas de los pacientes sobre el perfil epidemiológico y las enfermedades prevalentes, y los datos de adherencia a la medicación se evaluaron mediante el cuestionario Morisky-Green. Se utilizó el software Micromedex® para verificar las posibles interacciones medicamentosas. Resultados: La población estuvo conformada por 121 personas atendidas en la unidad de salud, predominantemente mujeres morenas $(83,6 \%$ y 46,3 respectivamente), con prevalencia de hipertensión arterial (65,7\%) y diabetes mellitus (40,3\%). Solo el 37,3\% de los pacientes mostró máxima adherencia a la medicación, principalmente por olvidos y descuidos en el uso de la medicación $(41,8 \%)$. El 37,9\% de los pacientes manifestó tener que pagar económicamente el tratamiento parcial o total, incluso con bajo poder adquisitivo ( $77,7 \%$ con 2 salarios mínimos o menos). La mayoría de los fármacos prescritos estaban relacionados con el sistema cardiovascular y el 20,9\% de las prescripciones tenían interacciones farmacológicas potenciales. Conclusión: La adherencia a la medicación fue intermedia para una población de bajos ingresos y educación media $(52,2 \%)$, con una alta prevalencia de enfermedades que afectan el sistema cardiovascular. Palabras clave: Adherencia a la medicación; Enfermedades cardiovasculares; Primeros auxilios; Estrategia de salud familiar.

\section{Introdução}

A Estratégia de Saúde da Família (ESF), implementada em 1994, como Programa Saúde da Família (PSF), hoje conta com mais de trinta mil equipes distribuídas por todo o território brasileiro (Sacchetim, 2012). Caracteriza-se como uma concepção de trabalho a partir da formação de vínculo entre os membros de uma equipe e a comunidade, diferentemente do modelo biomédico tradicional, e assim permite maior diversidade das ações de saúde nos territórios (MSB, 2000). A relação de trabalho é fundamentada na interdisciplinaridade e requer uma abordagem que estimule a constante comunicação horizontal entre os componentes da equipe, almejando assim, melhoria nas abordagens individual, da família e da comunidade, para que ocorra a efetiva implantação de um novo modelo de atenção à saúde.

A ESF tem papel fundamental no primeiro contato, na longitudinalidade e na coordenação do cuidado, devendo operar como base de estruturação das redes de atenção, com suporte dos serviços de apoio ao diagnóstico, assistência especializada e hospitalar. Entretanto, a USF não pode ser apenas um local de triagem e encaminhamento, necessita ser resolutiva, com profissionais capazes de assistir aos problemas de saúde mais prevalentes na população e de manejar novos saberes que, por meio de processos educativos, promovam a saúde e previnam agravos em geral, principalmente as de caráter crônico (Giovanella; Mendonça, 2008).

Neste sentido, o acompanhamento farmacológico dos pacientes com comorbidades especialmente as Doenças Crônicas Não Transmissíveis (DCNT's) se configura como relevante para os cuidados em saúde. Assim, a adesão ao tratamento medicamentoso pode ser compreendida como o grau de coincidência entre o comportamento do usuário e a recomendação do profissional de saúde perante o regime terapêutico (WHO, 2013).

Para mensurar a adesão à farmacoterapia, existem métodos diretos, tais como, dosagem do princípio ativo; e indiretos, por exemplo, contagem de comprimidos e o relato do usuário. O uso de instrumentos, como os questionários validados, permite mensurar a adesão com certa precisão sobre esse dado subjetivo a partir dos relatos do usuário. Uma das ferramentas mais utilizadas para avaliar a adesão é o Teste de Morisky-Green (TMG). A ferramenta apresenta como principais vantagens o reduzido número de questões e a fácil compreensão por parte do paciente (Borges et al., 2012).

Dentre as doenças que se faz necessário o uso crônico de medicamentos e consequentemente uma boa adesão 
medicamentosa, estão as Doenças Cardiovasculares (DCV) que se configuram como a primeira causa de morte no Brasil, consideradas graves problemas de saúde pública e apresentando alto gasto público por parte do Sistema Único de Saúde (SUS) (Guedes et al., 2016). Vários são os fatores de risco para essas doenças, tais como: tabagismo, dislipidemias, Hipertensão Arterial (HA), Diabetes Mellitus (DM), sobrepeso/obesidade, sedentarismo, alimentação pobre em vegetais e frutas, uso de álcool, estresse psicossocial, idade acima de 45 anos para homens e 55 anos para mulheres, de maneira que, muitas dessas condições são sensíveis à atenção básica (MSB, 2016a)

Sendo assim, o objetivo desse estudo foi avaliar a adesão medicamentosa das pessoas atendidas na Unidade de Saúde da Família em uma capital do nordeste do Brasil.

\section{Metodologia}

Foi realizado um estudo transversal, observacional, descritivo em uma Unidade de Saúde da Família localizada em Salvador, Bahia, nordeste do Brasil. A unidade é composta por quatro equipes de saúde da família que atendem a população adstrita no território para desenvolver as ações de saúde. Quanto a natureza dos dados da pesquisa, trata-se de um estudo quantitativo como descrito por Pereira et al., (2018).

Os dados utilizados constaram das informações, em prontuário, dos pacientes atendidos pela USF, no período entre janeiro de 2017 e janeiro de 2019, que foi a faixa temporal de atuação da equipe multiprofissional na Unidade de Saúde da Família. Como instrumento de coleta utilizou-se o teste de Morisky-Green (TMG) aplicado durante atendimento dos pacientes pela equipe multiprofissional. A versão do TMG utilizada constou das seguintes perguntas: 1) Você às vezes tem problemas em se lembrar de tomar a sua medicação? 2) Você às vezes se descuida de tomar seu medicamento? 3) Quando está se sentindo melhor, você às vezes para de tomar seu medicamento? 4) Às vezes, se você se sentir pior ao tomar a medicação, você para de tomá-la? O questionário totalizando ' 0 " foi considerado como máxima adesão medicamentosa, "'1-2" adesão medicamentosa moderada e "3-4" como baixa adesão medicamentosa.

A amostragem foi constituída por técnica não probabilística, por conveniência, dispondo do número de pacientes atendidos pela equipe multiprofissional da Unidade de Saúde da Família no período estudado. Foram incluídos, todos os pacientes atendidos pela equipe multiprofissional que faziam uso contínuo e/ou possuíam prescrição de pelo menos um medicamento, no mínimo seis meses. Foram excluídos os pacientes que não apresentaram no prontuário todas as informações sobre adesão medicamentosa.

Dispondo do programa EPI INFO, foram avaliadas variáveis demográficas, socioeconômicas, estilo de vida, doenças prevalentes, informações referentes aos medicamentos prescritos e como o paciente relatava se sentir referente ao seu estado de saúde. O software Micromedex ${ }^{\circledR}$ também foi utilizado para avaliar potenciais interações medicamentosas na prescrição. Foram analisados os pacientes que apresentaram interações medicamentosas, em suas prescrições, classificadas como grave ou contraindicada com presença de potencial interação medicamentosa. Por fim, foi utilizada a classificação "Anatomical Therapeutic Chemical Code" (ATC), realizada dispondo do site: https://www.whocc.no/atc_ddd_index_and_guidelines/atc_ddd_index/.

O trabalho seguiu todos os critérios da Ética em Pesquisa com Seres Humanos. Foi preservado o anonimato dos pacientes e conservando a autonomia. Para garantir o respeito à bioética e o cumprimento das medidas legais, o estudo foi submetido e aprovado pelo Comitê de Ética da Universidade do Estado da Bahia, sob o parecer de número: 3.502.164.

\section{Resultados}

Foram identificados 121 usuários do serviço de saúde e dentre os quais10 (8,2\%), não faziam uso de medicamentos, de forma que, foram excluídos do estudo. Obedecendo aos critérios de exclusão, foi constatado que dentre os atendimento restantes, 44 (36,3\%) não apresentavam o questionário Morisky-Green preenchido, dessa forma, também foram excluídos do 
estudo. Sendo assim, 67 pacientes preencheram os critérios de inclusão convergindo na inserção no estudo.

De acordo a Tabela 1 evidenciou-se que a maioria da população foi composta por pacientes do sexo feminino 83,6\%, adultas $55,2 \%$, casadas $49,3 \%$ e pardas $46,3 \%$. Observou-se que 55,2\% indivíduos possuíam IMC acima de 30 , sendo classificados como obesos. Acrescido a isso, outros 22,4\% dos pacientes apresentaram IMC entre 25-29,9, configurando-se como sobrepeso. Quando observados ocupação, renda e escolaridade; evidenciou-se que a maioria da população possuía alguma fonte de renda, sendo que 32,8\% estavam empregados e $17(28,2 \%)$ possuíam pensão ou aposentadoria. Por fim, renda familiar de 47,8\% foi estimada em até um salário mínimo 23,9\% entre um e dois salários mínimo e a escolaridade 52,2\% dos inclusos no estudo estudaram até o ensino médio.

Com finalidade de descrever os hábitos de vida e o estado geral de saúde, o etilismo foi referido por 35,8\% dos pacientes e o tabagismo 20,9\%. Outra variável levada em consideração foi a autopercepção da saúde, sendo que 49,2\% dos pacientes se autoreferiram doentes. Todos os dados citados acima são descritos na Tabela 1.

Tabela 1 - Perfil socioeconômico, condições de habitação e hábitos de vida de pacientes atendidos na Unidade de Saúde da Família, município de Salvador, Bahia.

\begin{tabular}{|c|c|c|}
\hline Variáveis & $\mathbf{N}$ & $\%$ \\
\hline \multicolumn{3}{|l|}{ Sexo } \\
\hline Masculino & 11 & 16,4 \\
\hline Feminino & 56 & 83,6 \\
\hline \multicolumn{3}{|l|}{ Cor } \\
\hline Preto & 19 & 28,4 \\
\hline Pardo & 31 & 46,3 \\
\hline Branco & 7 & 10,4 \\
\hline Amarelo & 1 & 1,5 \\
\hline Outros & - & - \\
\hline Não referido & 9 & 13,4 \\
\hline \multicolumn{3}{|l|}{ Faixa etária } \\
\hline Jovens (Até 19 anos) & 4 & 6,0 \\
\hline Adultos (20-59 anos) & 37 & 55,2 \\
\hline Idosos (>60 anos) & 26 & 38,8 \\
\hline \multicolumn{3}{|l|}{ IMC } \\
\hline Desnutrição $(<18,5)$ & 1 & 1,5 \\
\hline Normal $(18,5-24,9)$ & 8 & 11,9 \\
\hline Sobrepeso $(25,0-29,9)$ & 15 & 22,4 \\
\hline Obesidade $(>30)$ & 37 & 55,2 \\
\hline Não aferido & 6 & 9,0 \\
\hline \multicolumn{3}{|l|}{ Estado civil } \\
\hline Solteiro & 21 & 31,3 \\
\hline Casado & 33 & 49,3 \\
\hline Viúvo & 11 & 16,4 \\
\hline Não referido & 2 & 3,0 \\
\hline
\end{tabular}

\section{Renda}


Research, Society and Development, v. 10, n. 2, e31310212604, 2021

(CC BY 4.0) | ISSN 2525-3409 | DOI: http://dx.doi.org/10.33448/rsd-v10i2.12604

Um salário mínimo $\quad 32 \quad 47,8$

$\begin{array}{lll}\text { Entre um e dois salários mínimos } & 16 & 23,9\end{array}$

$\begin{array}{lll}\text { Acima de dois salários mínimos } & 8 & 11,9\end{array}$

\begin{tabular}{|c|c|c|}
\hline Não referido & 7 & 10,4 \\
\hline \multicolumn{3}{|l|}{ Ocupação } \\
\hline Desempregado & 20 & 29,9 \\
\hline Empregado & 22 & 32,8 \\
\hline Pensionista/aposentado & 17 & 25,4 \\
\hline Não referido & 8 & 11,9 \\
\hline \multicolumn{3}{|l|}{ Escolaridade } \\
\hline Fundamental & 23 & 34,3 \\
\hline Médio & 35 & 52,2 \\
\hline Superior & 2 & 3,0 \\
\hline Analfabeto & 5 & 7,5 \\
\hline Não referido & 2 & 3,0 \\
\hline \multicolumn{3}{|l|}{ Etilismo } \\
\hline $\operatorname{Sim}$ & 24 & 35,8 \\
\hline Não & 43 & 64,2 \\
\hline \multicolumn{3}{|l|}{ Tabagismo } \\
\hline Sim & 14 & 20,9 \\
\hline Não & 52 & 77,6 \\
\hline Não referido & 1 & 1,5 \\
\hline \multicolumn{3}{|c|}{ Autopercepção sobre a saúde } \\
\hline Saudável & 30 & 44,8 \\
\hline Doente & 33 & 49,2 \\
\hline Não referido & 4 & 6,0 \\
\hline
\end{tabular}

Fonte: Autores.

Dentre as principais doenças que acometem a população estudada, foi possível observar que o problema de saúde mais prevalente foi a hipertensão arterial, presente em 65,7\% da população estudada (homens:72,7\% e mulheres: 33,9 ). A segunda doença mais prevalente foi a diabetes mellitus afetando 40,3\% da população (Homens: $72,7 \%$ e mulheres: $33,9 \%$ ). Logo em seguida, a dislipidemia apresentou prevalência de 14,9\% (mulheres: 12,5\% e homens 27,2\%). Os dados supracitados estão descritos na Tabela 2. 
Tabela 2. Principais doenças encontradas no estudo por gênero na população atendida pela Unidade de Saúde da Família, município de Salvador, Bahia.

\begin{tabular}{|c|c|c|c|}
\hline Morbidades & Presente & Ausente & Prevalência (\%) \\
\hline \multicolumn{4}{|c|}{ Diabetes Mellitus } \\
\hline Homens & 8 & 3 & 72,7 \\
\hline Mulheres & 19 & 37 & 33,9 \\
\hline Total & 27 & 39 & 40,3 \\
\hline \multicolumn{4}{|c|}{ Hipertensão arterial } \\
\hline Homens & 9 & 2 & 81,9 \\
\hline Mulheres & 35 & 21 & 62,5 \\
\hline Total & 44 & 23 & 65,7 \\
\hline \multicolumn{4}{|l|}{ Dislipidemia } \\
\hline Homens & 3 & 8 & 27,2 \\
\hline Mulheres & 7 & 49 & 12,5 \\
\hline Total & 10 & 57 & 14,9 \\
\hline \multicolumn{4}{|l|}{ Depressão } \\
\hline Homens & 1 & 10 & 9,1 \\
\hline Mulheres & 2 & 54 & 3,6 \\
\hline Total & 3 & 64 & 4,4 \\
\hline \multicolumn{4}{|l|}{ Enxaqueca } \\
\hline Homens & - & 11 & - \\
\hline Mulheres & 2 & 54 & 3,6 \\
\hline Total & 2 & 54 & 3 \\
\hline
\end{tabular}

Fonte: Autores.

Após de realização da classificação ATC dos medicamentos nas prescrições, observou-se que dos 15 medicamentos mais frequentemente utilizados, 7 estavam na classificação " $\mathrm{C}$ ", referentes ao sistema cardiovascular. A losartana foi o medicamento mais prescrito 34 (50,8\%), seguida pela hidroclototiazida22 (32,8\%), metformina $22(32,8 \%)$, e sinvastatina 19 $(28,3 \%)$ como demonstra a Tabela 3. 
Tabela 3. Principais medicamentos de acordo a classificação ATC utilizados pelos pacientes atendidos na Unidade de Saúde da Família, município de Salvador, Bahia.

\begin{tabular}{cccc}
\hline Medicamentos & N & \% & Código ATC \\
\hline Losartana & 34 & 50,8 & C09CA01 \\
Hidroclorotiazida & 22 & 32,8 & C03AA03 \\
Metformina & 22 & 32,8 & A10BA02 \\
Sinvastatina & 19 & 28,3 & C10AA01 \\
Ácido Acetilsalicílico & 16 & 23,8 & N02BA01 \\
Insulina NPH & 11 & 16,4 & A10AE01 \\
Anlodipino & 7 & 10,4 & C08CA01 \\
Glibenclamida & 5 & 7,4 & A10BB01 \\
Omeprazol & 7 & 10,4 & A02BC01 \\
Glicazida & 7 & 10,4 & A10BB09 \\
Enalapril & 4 & 6 & C09AA02 \\
Dipirona & 4 & 6 & N02BB02 \\
Sulfato ferroso & 5 & 7,4 & B03AA07 \\
Clonidina & 4 & 6 & C02AC01 \\
Nifedipino & 1 & 1,5 & C08CA05 \\
\hline
\end{tabular}

Fonte: Autores.

Observou-se que a maioria da população estudada apresentou adesão moderada ao tratamento 44,8\%. Apesar da USF ter sido responsável pela dispensação de medicamentos para 53\% da população, 37,9\% dos pacientes referiu adquiri-los, dependendo parcialmente ou totalmente de recursos próprios.Por fim, o tipo de medicamento mais utilizado foram os genéricos $71,2 \%$. Quando analisado o número de medicamentos utilizado pelos pacientes encontrou-se que $73,1 \%$ utilizavam menos de cinco medicamentos. Ademais, foram observadas potenciais interações medicamentosas nas prescrições de 14 pacientes (20,9\%), como exposto na Tabela 4. 
Tabela 4. Adesão medicamentosa, quantidade, tipo, forma e local de aquisição dos medicamentos pelos pacientes atendidos na Unidade de Saúde da Família e potenciais interações medicamentosas encontradas.

\begin{tabular}{|c|c|c|}
\hline Variáveis & $\mathbf{n}$ & $\%$ \\
\hline \multicolumn{3}{|l|}{ Adesão medicamentosa } \\
\hline Baixa adesão & 12 & 17,9 \\
\hline Adesão Moderada & 30 & 44,8 \\
\hline Máxima adesão & 25 & 37,3 \\
\hline \multicolumn{3}{|l|}{ Local de aquisição do medicamento } \\
\hline USF & 35 & 53 \\
\hline Drogaria & 13 & 19,7 \\
\hline Aqui tem farmácia popular & 2 & 3,0 \\
\hline Drogaria + USF & 12 & 18,2 \\
\hline Aqui tem farmácia popular + USF & 4 & 6,1 \\
\hline \multicolumn{3}{|l|}{ Tipo de medicamento } \\
\hline Genérico & 47 & 71,2 \\
\hline Referência & 8 & 12,2 \\
\hline Similar & 2 & 3,0 \\
\hline Genérico + Referência & 7 & 10,6 \\
\hline Similar + Genérico & 2 & 3,0 \\
\hline \multicolumn{3}{|l|}{ Quantidade de medicamentos em uso } \\
\hline Até 4 medicamentos & 49 & 73,1 \\
\hline 5 ou mais medicamentos (Polifarmácia) & 18 & 26,9 \\
\hline \multicolumn{3}{|l|}{ Potenciais interações medicamentosas } \\
\hline Sim & 14 & 20,9 \\
\hline Não & 53 & 79,1 \\
\hline
\end{tabular}

Fonte: Autores.

Quando avaliados individualmente as limitações para adesão à terapia medicamentosa, descritas como perguntas no questionário de Morisky-Green, foi observado que esquecer de tomar os medicamentos e descuidos quanto a terapêutica foram descritas por $41,8 \%$ dos pacientes; $26,9 \%$ dos pacientes referiram interromper o uso dos medicamentos quando se sentiram pior e $20,9 \%$ dos pacientes referiram interromper o tratamento quando se sentem melhor. Os dados são descritos na Tabela 5.

Tabela 5. Principais limitações para adesão ao tratamento medicamentoso pelos pacientes atendidos na Unidade de Saúde da Família, município de Salvador, Bahia.

\begin{tabular}{ccc}
\hline Variáveis & $\mathbf{n}$ & $\boldsymbol{\%}$ \\
\hline Problemas em lembrar-se de tomar o medicamento & 28 & 41,8 \\
Descuido ao tomar o medicamento & 28 & 41,8 \\
Para de tomar o medicamento quando se sente pior & 18 & 26,9 \\
Para de tomar o medicamento quando se sente melhor & 14 & 20,9 \\
\hline
\end{tabular}




\section{Discussão}

A prevalência das DCV's, a utilização de medicamentos e adesão medicamentosa foram achados demonstrados neste estudo. Desse modo, segundo a Organização Panamericana de Saúde, as doenças cardiovasculares representam a principal causa de mortalidade no Brasil e no mundo (OPAS, 2017). Neste sentido, faz-se necessário uma boa adesão ao tratamento medicamentoso para melhorar a qualidade de vida, prevenir agravos e o óbito (Pesinato et al.,2015). Em contraponto, a não adesão ao tratamento medicamentoso, variando de 15 a 93\% para portadores de doenças crônicas, com média estimada em $50 \%$, é a principal responsável por falhas no tratamento e uso irracional de medicamentos, sendo considerada como um problema de saúde pública, e denominada como "epidemia invisível" (MSB, 2016b).

A população do estudo foi composta, predominantemente, por mulheres, pardas adultas, apresentando sobrepeso ou em estado de obesidade. Em relação a raça/cor o valor corrobora com a Pesquisa Nacional por Amostra de Domicílios Contínua (PNAD) no ano de 2016, na qual, os pardos representavam a maioria $(46,7 \%)$ da população brasileira. Um fator observado no estudo foi a baixa presença masculina. Outros estudos (Oliveira et al., 2015; Moreira; Fontes; Barboza, 2012; Machin et al.2011) ressaltaram as limitações nos programas de atenção específica aos homens; baixa autonomia do homem quanto ao cuidado da saúde; visão masculina de que as unidades básicas de saúde são voltadas às mulheres, crianças e idosos além de limitações dos profissionais em captá-los, dificultam a presença dos homens nos serviços de saúde.

Além disso, a população estudada possuía fonte de renda entre 1 e 2 salários mínimos, eram casadas, com nível de escolaridade até o ensino médio. Poucos indivíduos recebiam mais de 2 salários mínimos, que não necessariamente se reflete em significante maior poder aquisitivo. O referido dado é um fator de risco à baixa adesão medicamentosa, visto que, o subgrupo de pessoas inseridas no mercado de trabalho, em especial aqueles trabalhadores não qualificados e com baixo poder aquisitivo (classes C, D e E) apresenta maior risco de não adesão (Sobral et al., 2017; Tavares et al., 2016; Di Matteo, 2004).

Associado a isso, é conhecido que as condições sociais interagem com as condições fisiológicas determinando a propensão às diferentes doenças (Melchior et al., 2016). São inúmeras as evidências na literatura de que a hipertensão arterial, as doenças cardiovasculares e a mortalidade decorrente dessas doenças são mais marcantes entre as pessoas de menor nível socioeconômico e de menor escolaridade (Martin et al., 2014). A prevalência de obesidade e sobrepeso encontrada no estudo foi bastante elevada. E estima-se que no Brasil 52,6\% dos homens e 44,7\% das mulheres, com mais de 18 anos, estão acima do peso ideal (Arruda et al., 2015). Tanto a obesidade quanto o sobrepeso constituem fatores de risco para a carga global de doenças cardiovasculares, diabetes, câncer de cólon, reto e de mama, cirrose, dentre outras (WHO, 2013). Ademais, doenças crônicas tais como diabetes, doenças cardíacas e hipertensão são associadas ao baixo grau educacional, e apresentam prevalência mais acentuada no gênero feminino em caráter de vulnerabilidade socioeconômica (Dalstra et al., 2005). De maneira que, existe uma relação entre subgrupos de pacientes com doenças crônicas e dificuldades de adesão ao tratamento medicamentoso (Di Matteo, 2004). Tavares e colaboradores (2016) observaram a prevalência de baixa adesão ao tratamento farmacológico de doenças crônicas associadas aos indivíduos residentes na região Nordeste e Centro-Oeste do país; que nunca estudaram; que tiveram que pagar parte do tratamento medicamentoso; com pior autopercepção da saúde; com três ou mais doenças; que referiam limitação causada por uma das doenças crônicas; e que faziam uso de cinco medicamentos ou mais Esses dados encontrados pelo autor dialogam com a população no presente estudo.

No que tange a polifarmácia, foi encontrado que cerca de um quarto da população fazia uso de cinco ou mais medicamentos. De maneira que, os medicamentos mais utilizados foram os que agem no sistema cardiovascular (grupo $\mathrm{C}$ da classificação ATC). A utilização de quantidades maiores de medicamentos geralmente está associada à idade maior que 45 anos (sendo uma prática ainda mais acentuada em indivíduos com mais de 65 anos), baixa autopercepção de saúde, presença de doenças crônicas, assistência privada à saúde, atendimento em serviço de emergência e região do país. Ademais, é observado que a polifarmácia tem papel importante na ocorrência de eventos adversos, na limitação da adesão medicamentosa e no custo 
dos cuidados com a saúde (Nascimento et al., 2017).

$\mathrm{Na}$ população estudada foram encontradas potenciais interações medicamentosas nas prescrições. Em geral as interações medicamentosas estavam relacionadas com ocorrência aumentada de potenciais reações adversas, tais como: miopatia, rabdomiólise, trombose e nefrotoxicidade, condições que podem afetar diretamente na adesão ao tratamento farmacológico, visto que, muitos indivíduos interrompem o tratamento farmacoterapêutico em casos de piora. O estudo de Santa-helena; Nemes; Neto (2010) demonstrou que na população de 595 pacientes atendidos por unidades de saúde da família de Blumenau, em uso de tratamento anti-hipertensivo, a interrupção prévia do tratamento estava associada com relato de reações adversas $(\mathrm{P}<0,01)$ e os indivíduos que referiram tais reações utilizavam mais medicamentos do que aqueles que não relataram $(5,2$ e 4,4 , respectivamente) $(\mathrm{P}<0,01)$.

Os "problemas em lembrar-se de tomar o medicamento" e "descuido ao tomar o medicamento" foram as causas mais limitantes à adesão medicamentosa, de maneira que, apenas um terço da população apresentava máxima adesão ao tratamento. Essas causas também foram apontados nos estudos de Sobral et al., (2017), Carvalho et al., (2012) e Santos; Oliveira; Colet (2010) quando aplicado o questionário de Morisky-Green. Um agravante encontrado nesses estudos foi que pelo menos metade dos pacientes desconheciam o nome do medicamento, dose administrada e não detinham conhecimento sobre o período de uso. Em outro estudo (Carvalho et al., 2012), foi possível observar que apenas 26,8\% dos pacientes apresentaram máxima adesão medicamentosa.

\section{Considerações Finais}

Observou-se que a população atendida pela Unidade de Saúde da Família em questão foi majoritariamente composta por mulheres, apresentava um perfil de vulnerabilidade para as doenças cardiovasculares e de média adesão medicamentosa, principalmente devido ao esquecimento e o descuido ao tomar o medicamento. As DCV's apresentaram alta prevalência, associado a isso, foi descrito que a obesidade e o sobrepeso são condições extremamente recorrentes e aparentemente não são reconhecidas como problemas de saúde. Ademais, mesmo com baixo poder aquisitivo, boa parte da população arcou financeiramente com parte ou totalidade de seu tratamento, por meio da aquisição em drogarias, o que dificulta a adesão medicamentosa. Agravando a situação, foi exposto que muitas das prescrições apresentavam potenciais interações medicamentosas o que pode limitar a adesão ao tratamento farmacoterapêutico. São necessários mais trabalhos avaliando adesão medicamentosa da população brasileira, dispondo de diferentes populações amostrais, com intuito de identificar possíveis fatores limitantes e traçar estratégias para superá-los.

\section{Referências}

Arruda, G. O., Santos, A. L., Teston, E. F., Cecilio, H. P. M., Radovanovic, C. A. T., \& Marcon, S. S. (2015). Associação entre autopercepção de saúde e características sociodemográficas com doenças cardiovasculares em indivíduos adultos. Rev Esc Enferm USP, 49(1):61-68.

Borges, J. W. P., Moreira, T. M. M., Rodrigues, M. T. P., \& Oliveira, C. J. (2012). Utilização de questionários validados para mensurar a adesão ao tratamento da hipertensão arterial: uma revisão integrativa. RevEscEnferm USP, 46(2):487-94.

Carvalho, A. L. M., Leopoldino, R. W. D., Silva, J. E. G., \& Cunha, C. P. (2012). Adesão ao tratamento medicamentoso em usuários cadastrados no Programa Hiperdia no município de Teresina (PI). Cien\& Saúde Col. 2012, 17(7):1885-1892.

Dalstra, J. A. A., Kunst, A. E., Borrell, C., Breeze, E., Cambois, E., Costa, G., Geurts , J. J. M., Lahelma, E., Van Oyen, H., Rasmussen, N. K., Regidor, E., Spadea, T., \& Mackenbach, J. P. (2005). Socioeconomic differences in the prevalence of common chronic diseases: an overview of eight European countries. Int Jour.of Epi. 34:316-326.

Di Matteo, M. R. (2004). Variations in patients adherence to medical recommendations: a quantitative review of 50 years of research. Med Care. $42: 200-209$.

Giovanella, L., \& Mendonça, M. H. M. (2008). Políticas e Sistemas de Saúde no Brasil. Rio de Janeiro. Editora Fiocruz. Cap 16, $575-625$.

Gomes, R., Moreira, M. C. N., Nascimento, E. F., Rebello, L. E. F. S., Couto, M. T., \& Schraiber, L. B. (2011). Os homens não vêm! Ausência e/ou invisibilidade masculina na atenção primária. Ciência \& Saúde Coletiva,16(Supl. 1):983-992. 
Guedes, R. F., Melo, T. E., Lima, A. P., Guimarães, A. L. A., Moreira, N. B. B., \& Garcia, P. G. (2016). Análise do perfil lipídico e dos fatores de risco associados a doenças cardiovasculares em acadêmicos da área da saúde. $H U$ Ver, 42(2): 159-164.

Machin, R., Couto, M. T., Silva, G. S. N., Schraiber, L. B., Gomes, R., Figueiredo, W. S.Valença, O. A., \& Pinheiro, T. F. (2011). Concepções de gênero, masculinidade e cuidados em saúde: estudo com profissionais de saúde da atenção primária. Ciência \& Saúde Coletiva. 16(11):4503-4512.

Martin, R. S. S., Godoy, I., Franco, R. J. S. F., Martin, L. C., \& Martins, A. S. (2014). Influência do nível socioeconômico sobre os fatores de risco cardiovascular. Jor bras de Enf. mar-abr,102 (2):34-37.

Melchior, M., Berkman, L. F., Kawachi, I., Krieger, N., Zins, M., Bonenfant, S., \& Goldberg, M.(2006). Lifelong socioeconomic trajectory and premature mortality (35-65 years) in France: findings from the GAZEL Cohort Study. Jor. Epi. Com Health. 60(11): 937-944.

Ministério da Saúde do Brasil (MSB). (2000). Cadernos de Atenção Básica Programa de Saúde da Família. Ministério da Saúde.

Ministério da Saúde (MSB). (2016a). Secretaria de Ciência, Tecnologia e Insumos Estratégicos. Departamento de Ciência e Tecnologia. Síntese de evidências para políticas de saúde: Adesão ao tratamento medicamentoso por pacientes portadores de doenças crônicas. Ministério da Saúde.

Ministério da Saúde (MSB). (2016b). Secretaria de Vigilância em Saúde. Departamento de Vigilância de Doenças e Agravos não Transmissíveis e Promoção da Saúde. Vigitel Brasil 2015: vigilância de fatores de risco e proteção para doenças crônicas por inquérito telefônico: estimativas sobre frequência e distribuição sociodemográfica de fatores de risco e proteção para doenças crônicas nas capitais dos 26 estados brasileiros e no Distrito Federal em 2015.1. Ed. Brasília: Ministério da Saúde.

Moreira, R. L. S., Fontes, W. D., \& Barboza, T. M. (2012). Dificuldades de inserção do homem na atenção básica a saúde: a fala dos enfermeiros. Esc Anna Nery. 18(4):615-621.

Nascimento, R. C. M., Álvares, J., Junior, A. A. G., Gomes, I. C., Silveira, M. R., Costa, E. A., Leite, S. N., Costa, K. S., Soeiro, O. M., Guibu, I. A., Margô Gomes de Oliveira Karnikowski, M. G. O., \& Acurcio, F. A. (2017). Polifarmácia: uma realidade na atenção primária do Sistema Único de Saúde. Rev Saude Publica, 51 Supl 2:1-12.

Oliveira, M. M., Daher, D. V., Silva, J. L. L., \& Andrade, S. S. C. A. (2015). A saúde do homem em questão: busca por atendimento na atenção básica de saúde. Ciência \& Saúde Coletiva. 20(1):273-278.

Organização Pan-americana de Saúde (OPAS). (2017). Doenças cardiovasculares [Internet]. Brasília: Organização Pan-americana de Saúde.

Pereira A. S., Shitsuka, D. M., Parreira, F. J., \& Shitsuka, R. (2018). Metodologia da pesquisa científica. UFSM.

Pesinato, R. M., Richards, G. L., Cintra, F. F., \& Tamassia, A. F. (2015). Adesão ao tratamento de pacientes com fatores de risco cardiovascular em ambulatório da Zona Sul de São Paulo. Rev Soc Bras Clin Med. 13(3):185-189.

Sacchetim, S. C. (2012). Comentários sobre o panorama atual da Atenção Primária à saúde no Brasil e sua interface com a formação em medicina de família e comunidade. Rev Edu em Saúde. Nov, 01(1).

Santa-helena, E. T., Nemes, M. I. B., \& Neto, J. E. (2010). Fatores associados à não-adesão ao tratamento com anti-hipertensivos em pessoas atendidas em unidades de saúde da família. Cad. Saúde Publica. Rio de Janeiro, dez,26(12):2389-2398.

Santos, F. S., Oliveira, K. R., \& Colet, C. F. (2010). Adesão ao tratamento medicamentoso pelos portadores de Diabetes Mellitus atendidos em uma Unidade Básica de Saúde no Município de Ijuí/RS: um estudo exploratório. Res Ciênc Farm. Básica Apl, 31(3):223-227.

Sobral, P. D., Oliveira, D. C., Gomes, E. T., Carvalho, P. O., Brito, N. M. T., Oliveira, D. A. G. C., Aragão, E. R., \& Godoi, E. T.(2017). Razões para não adesão a fármacos em pacientes com doença arterial coronariana. Rev Soc Bras Clin Med. Jul- set,15(3):166-70.

Tavares, N. U. L., Bertoldi, A. D., Mengue, S. S., Arrias, P. S. A., Luiza, V. L., Oliveira, M. A., Ramos, L. R., Farias, M. R., \& Dal Pizzo, T. S. (2016). Fatores associados à baixa adesão ao tratamento farmacológico de doenças crônicas no Brasil. Rev Saúde Púb. 50(supl 2):1-11.

World Health Organization (WHO). (2013). Adherence to long term therapies: evidence for action. [Internet]. Organização Mundial de Saúde, Geneva. 